\title{
On the Convergence of Financial Distress Propagation on Generic Networks
}

\author{
Irene UNCETA ${ }^{\mathrm{a}, 1}$, Bernat SALBANYA ${ }^{\mathrm{a}}$ and Jordi NIN ${ }^{\mathrm{a}}$ \\ ${ }^{a}$ Universitat Ramon Llull, ESADE, Barcelona
}

\begin{abstract}
.
Financial networks represent the daily business interactions of customers and suppliers. Research in this domain has mainly focused on characterizing different network structures and studying dynamical processes over them. These two aspects, structure and dynamics, play a key role in understanding how emergent collective behaviors, such as those that arise during economic crises, propagate through networks. Business interactions between companies form a direct and weighted network, where the financial distress of a node depends on the ability of its customers to fulfill payments. In situations where there is no such inbound cash flow, a company may have to close down due to a lack of liquidity. Interconnection therefore seems to be at the core of systemic fragility. Whether the nature and form of this connection may have an impact on how distress is propagated is still an open question. In this paper, we study how disruptive events propagate through different network structures, under different scenarios. For this purpose, we use a liquidity model that describes how the economy of nodes evolves from a given initial state in terms of their interactions. From our experiments, we empirically conclude that most of the studied network dynamics reach a steady-state, even in the presence of large noise values.
\end{abstract}

Keywords. Non-linear systems, financial networks, convergence testing

\section{Introduction}

In a customer-supplier network, nodes refer to individual firms and connections map their interactions or economic exchanges. This representation has allowed researchers to study how information, money, materials or components can flow through networks of different shapes and structures [1]. In the case of financial markets, a network approach has been particularly successful in characterizing the dynamics of customer-supply networks. These networks play a fundamental role in the optimization of production chains and the analysis of systemic risk [2]. Hence, studying the dynamics of these networks is of outermost importance to predict emergent phenomena that could potentially have negative consequences on individual nodes, as well as on the economy as a whole [3]. Even small disturbances can drive supply networks away from their desired state and towards unstable regimes [4] where disruptions are amplified through the network [5].

\footnotetext{
${ }^{1}$ Corresponding Author: Irene Unceta, Department of Operations, Innovation and Data Science, Universitat Ramon Llull, ESADE, Barcelona; E-mail: irene.unceta@esade.edu.
} 
Financial distress propagation has been studied using different models and network structures [6]. Recent studies have shown that there exist significant correlations between the local topological properties of nodes in a financial network and their risk of default [7]. Other studies have focused on understanding how this risk is propagated through the network, leading to scenarios of systemic instability. Or, even, on understanding how a node's economy state evolves in time.

In particular, in [8] authors introduced an additive economic model that described how node liquidity evolves in time using an equation that captures both exchanges between individuals and random speculative trading. This model has been assayed on a synthetic, heterogeneous (power-law) network. In this type of network, some nodes are highly connected, i.e. they have many links to other nodes. Yet, the overall number of connections is low. As a result, phenomena such as preferential attachment emerge, whereby new nodes tend to connect to nodes with larger degrees, i.e. hubs. In line with these property, authors observed that a few agents tended to concentrate the majority of the wealth, following a Pareto-tail distribution. This conclusion was drawn by using a simple but effective equation where trading was introduced as two independent additive noises over time. This model has been then further refined and adapted to study distress propagation in [9]. In this follow-up work, the authors have shown that the proposed model reaches a steady-state even in the presence of large noise volatility values, when the system is composed of a single agent and a market node.

In this paper, we build on the intuition introduced in these two papers to evaluate the convergence of the described model for different network architectures. To this end, we expose a synthetic network, with initially given liquidity conditions, to specific disruption events, and simulate the evolution of the liquidity distribution until its convergence. Notably, we study the evolution of the liquidity distribution for different generic network topologies, in different scenarios that may or may not include a market node. From our experiments, it is possible to extract meaningful insights about how the topology affects distress propagation and what the impact of a market node is for ensuring resilience against disruption.

\section{Materials and methods}

Time evolution of a financial network can be monitored by modelling the interactions between the nodes at each time step and updating each node's state accordingly. The state of a node can be related to any given property. For example, its liquidity.

\subsection{Liquidity model}

Following [8], we can define the liquidity $L(t)$ of a node as its amount of disposable money. The liquidity of a node has a direct relationship with two different elements: the economic exchanges between the considered node and the other nodes in the network, and the market volatility. The economic exchanges are usually represented by the edges of the considered network. The volatility, in turn, accounts for the uncertainty of the market, and is defined as an external factor affecting each node independently. We can approximate it by means of a Gaussian distribution.

To simulate the evolution of the liquidity over time for individual nodes, we introduce a liquidity model that describes how the economic activity of each node evolves in 
terms of its interactions with other nodes, given an initial state. This initial state $L(0)$ stands for the initial liquidity conditions of each node at time 0 . The larger its value, the more money the considered node will dispose of to face potential economic volatility, crises, and changes in the money flows through the network. Given this initial state, we can compute the liquidity $L_{i}(t)$ of a node $i$ at any given point in time $t$ as:

$$
L_{i}(t+1)=L_{i}(t)(1+\eta(0, \sigma))+\sum_{j \in N_{i}} w_{j i} P(t) H\left(L_{j}(t)-w_{j i}\right)-\sum_{j \in N_{i}} w_{i j} P(t) H\left(L_{i}(t)-w_{i j}\right)
$$

where $\eta(0, \sigma)$ stands for the economic volatility represented as a Gaussian noise with mean 0 and standard deviation $\sigma, N_{i}$ stands for the set of neighbors of node $i, w_{j i}$ and $w_{i k}$ correspond to the weights of an edge from $j$ to $i$ and vice versa, $P(t)$ is a random variable which follows a Bernoulli distribution with probability $p$ and, finally, $H\left(L_{i}(t)-\right.$ $w_{i j}$ ) represents a Heaviside step function which evaluates to 1 if the node $i$ has enough liquidity to afford the considered money transaction and 0 otherwise.

A Gaussian distribution governs market volatility for each node. Following the parameters of this distribution, a random normal value is given to every single node for each time-step simulation. Its impact on the liquidity is proportional to the liquidity at the previous time step and may affect the outcome positively or negatively. For this reason, the mean of the Gaussian distribution is set to zero $(\mu=0)$.

The money exchanges are, in turn, controlled by the global parameter $p$, corresponding to the probability of a Bernoulli distribution. The value of $p$ ranges from 0 to 1 . When $p=1$, the network is fully activated, meaning that all money exchanges take place. For lower values of $p$, the network is not fully activated, i.e., certain due payments never take place. Therefore, $p$ can be understood as the fraction of active exchanges at any time.

The equation above allows us to simulate the evolution of a given network in time under different initial conditions, encoded by the initial state $L_{i}(0)$ for each node. Depending on the considered network structure, we can study the effect of these conditions in the overall state of the network by the time we reach convergence. In [9], authors have shown that a single node network following this model reaches a steady-state even in the presence of large noise volatility values. For that network, the interplay between the outbalance of the initial income and outcome money flows, and the multiplicative noise level $\sigma$ leads to a symmetry in the final liquidity distribution. Here, we extend this single node model system to more complex scenarios and assay this model in under different economic conditions. These conditions are defined by different initial states and activation levels of the network. For each set of conditions we simulate the evolution of liquidity in time, and verify that the proposed model converges to a stationary state.

\subsection{Scenarios}

In some cases we introduce a market node. This node accounts for unknown transactions between the nodes of the network, i.e. for economic transactions conducted elsewhere. Including these data is important to obtain a reliable approximation of the economic state of each node in time. Combined with a large activation of the system, which means nodes are capable of doing exchanges, a market node decreases the overall distress probability of the considered systems. 
In our experiments we have assumed that the market node has infinite liquidity and that it transfers money to the other nodes with a liquidity ratio equal to 1.5 . In practice, this means that for a full activation of the network $(p=1)$, individual nodes earn 1.5 from their connections, including the market node, and pay back 1 . Therefore, in each timestep, profits are set to 0.5. Considering the amount of taxes and benefits that each node must pay and earn, we consider this to be a realistic value [9]. Moreover, this allows us to simulate economic exchanges within a growing economic context and avoids a general default of the system, which is seldom observed in practice.

The first scenario we consider is a Star network. This network is an extension of the single node network, where the different nodes are connected forming a star, as shown in Figure. 1 (a). In this structure, each $N_{i}$ node is linked to the market node $N_{0}$, and is independent of the remaining nodes $N_{j}$ for $j \neq i$, meaning that there is no exchange of money among them. The market node provides liquidity to nodes and receives the payments from it.

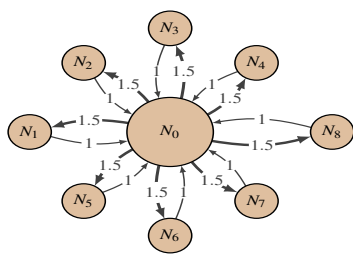

(a)

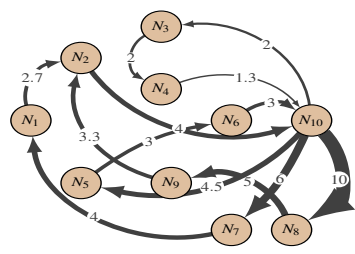

(b)

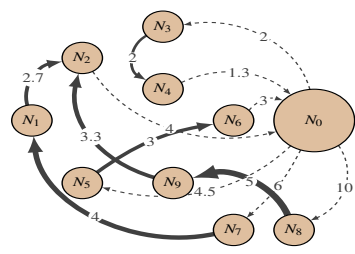

(c)

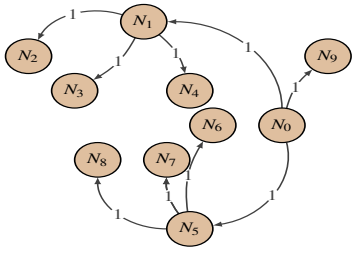

(d)

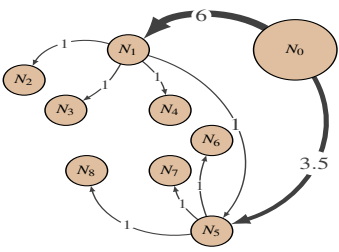

(e)

Figure 1. Topology of the simulated networks: (a) Star, (b) Erdos-Renyi without market node, (c) Erdos-Renyi with market node, (d) Power Law without market node, (e) Power Law with market node.

A more complex monetary exchange is that of an Erdos-Renyi network, where there exist random edges among the nodes. In this configuration, any node $N_{i}$ is linked with the same probability to the other nodes. Each edge is included in the network with a certain probability, which is independent for each edge. Therefore, each node is statistically independent of the rest of $N_{j}$ nodes for $j \neq i$. This structure represents a collaborative economy, where money flows through the agents. In this paper we study this network first in absence of a market node, as shown in Figure. 1 (b). We later introduce this market node as displayed in Figure. 1 (c) and evaluate its impact on the overall dynamics. When having the market node $N_{0}$, we assume all the remaining nodes are linked to it.

Finally, a more realistic scenario is that of a Power Law network. This type of network replicates an economic structure that includes both prevalent and secondary companies, as generally observed in real markets. Some nodes $N_{i}$ are highly connected, meaning they have many links to other $N_{j}$ nodes for $j \neq i$. Yet, the overall number of connections among all nodes is low. We can understand this configuration as representing 
a capitalist economy. Similarly to the Erdos-Renyi case, we consider this network with and without a market node. In the former case, all nodes are connected to the market node $N_{0}$ to keep a constant liquidity ratio.

We have created synthetic networks according to the five scenarios described above. In what follows, we study the convergence of each system's liquidity and analyse how disruptive events propagate through the different network topologies.

\section{Numerical Simulations on Generic Networks}

For the case of the Star network, we have created simulated scenarios with 100 nodes. For both the Erdos-Renyi and the Power-law configurations, we have included up to 1,000 nodes. For the latter case, each new node adds three random edges, with a probability of adding a new triangle after adding an edge of $30 \%$.

For each of the five configurations, we have conducted 1,000 simulations during 5,000 time-steps for a volatility $\sigma=0.05$ and $\sigma=0.3$. These values are chosen following [9] in order to represent two scenarios: low and high volatility. Or in other words, stable and unstable economies. We have assumed every single node to have enough liquidity to withdraw the first installment as an initial state. During each time step, we have simulated money exchanges according to the Bernoulli probabilities associated with the different nodes and computed their liquidity in time. Throughout the simulations, we have allowed nodes to be indebted and to recover from this state.

\subsection{Discussion of results}

First, we evaluate the convergence of the median node liquidity in time for different values of the Bernoulli probability $p$. In most cases, we observe a steady-state around 5,000 time-steps, when the median of the liquidity stabilizes. We also study the impact of disruptions for the networks described in the previous section by analyzing the liquidity distribution at the steady-state for each Bernoulli probability $p$, across 1,000 simulations.

\subsubsection{Star Network}

In order to analyze the effect of a central market node ruling the economy, we now study the evolution of the liquidity for the Star network scenario. For both $\sigma=0.05$ and $\sigma=0.3$, we observe that the median of the liquidity tends to a steady state, as shown in Figure. 2 (a) and (d), for the different values of the Bernoulli probability $p$. Depending on the value of $p$, we observe different growth rates of the median liquidity. For this network, the market node supplies liquidity every time-step to the individual nodes. However, this growth rate depends on the activation of the system. Thus, for higher activation values the median liquidity is greater than for low activations.

As observed for the previous case, the liquidity reaches a steady state for any value of $p$. The speed with which this steady state is reached depends on the volatility. For $\sigma=0.05$, the system reaches the steady state around $t=5,000$, whereas for $\sigma=0.3$ this happens around $t=200$. Moreover, for this case, we observe a decrease for lower activation rates. This is due to the greater market shocks that, combined with fewer transactions, make nodes more susceptible to falling into indebtedness. 


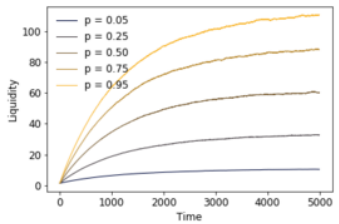

(a) $\sigma=0.05$

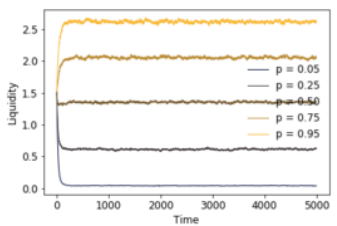

(d) $\sigma=0.3$

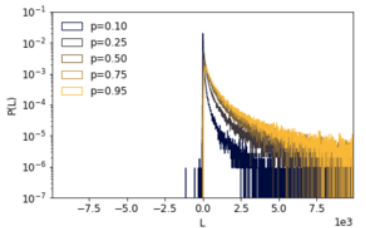

(b) $\sigma=0.05$

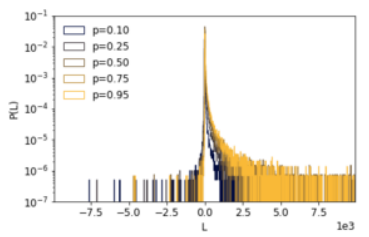

(e) $\sigma=0.3$

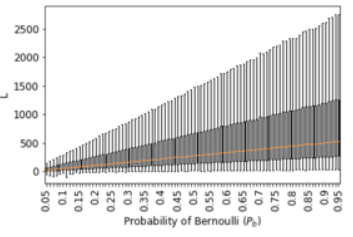

(c) $\sigma=0.05$

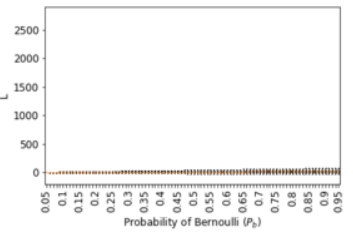

(f) $\sigma=0.3$

Figure 2. (a)(d) Median of the total liquidity at time $t$ for different combinations of Bernoulli probability $p$ for a Star network with market. (b)(e) Distribution of the system liquidity at time $T=5,000$. Note that, y-scale is logarithmic. (e)(f) Distribution of the system liquidity at time $T=5,000$.

Figures. 2 (b) and (e) show the distributions of the core system liquidity at the steady state. The liquidity distributions are not symmetric for any values of $\sigma$ and $p$, although we still observe a peak at $t=5,000$. The peak increases and the tails flatten as volatility increases, having more portion of nodes with debts in this case.

In Figure. 2 (c) and (f), where the boxplots display the different liquidity values at the steady state across the different values of $p$, we also observe the asymmetry in the liquidity distribution and the growth in the liquidity median. The injection of credit by the market node and a major activation shape the liquidity distribution. A smaller spread for high volatility is a consequence of greater market shocks.

\subsubsection{Erdos-Renyi Network}

We can represent a collaborative economy by means of an Erdos-Renyi network. In the absence of a market node, the behavior of the liquidity is completely random, and it does not converge to a steady state, as we can observe in Figures. 3 (a) and (d), where the median liquidity values are plotted in time for volatility $\sigma=0.05$ and $\sigma=0.3$. These show that an Erdos-Renyi without a market acts as a random and isolated system.

In Figures. 3.(b) and (e), the distributions of the core system liquidity at the steady state are displayed. We observe a symmetry in the liquidity distribution at time $t=5,000$. That is, for higher volatility, the peak of the distribution increases, whereas the tails have a lower spread. Moreover, some differences are observed in the liquidity boxplots for different $p$ at the steady state depicted in Figures. 3.(c) and (f). Here, for $\sigma=0.05$, the maximum spread of the liquidity is for the greatest activation of the system $(p=1)$. Yet for $\sigma=0.3$, the maximum swifts to $p=0.5$.

Significant differences are observed when a market node is added to the Erdos-Renyi network. Figures. 2 (a) and (d) show that the median of the liquidity tends to a steady state for both $\sigma=0.05$ and $\sigma=0.3$ and the different values of the Bernoulli probability $p$. Again, the presence of a market node has an effect on the evolution of liquidity, with different growth rates for the different values of $p$, as observed for the Star network. 


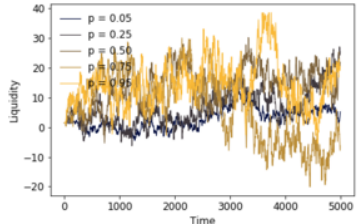

(a) $\sigma=0.05$

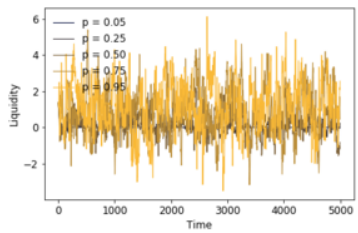

(d) $\sigma=0.3$

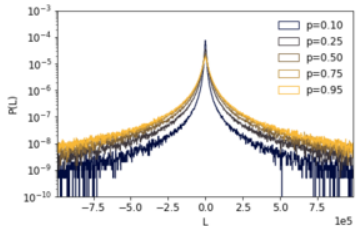

(b) $\sigma=0.05$

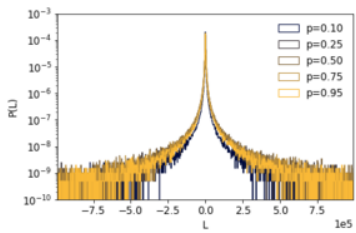

(e) $\sigma=0.3$

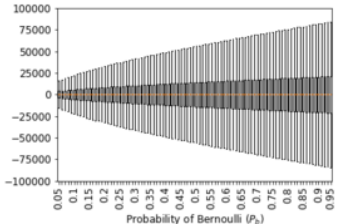

(c) $\sigma=0.05$

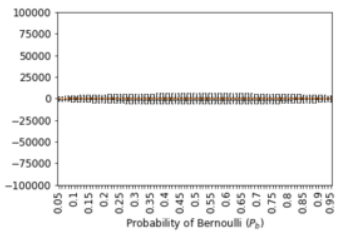

(f) $\sigma=0.3$

Figure 3. (a)(d) Median of the total liquidity at time $t$ for different combinations of Bernoulli probability $p$ for an Erdos-Renyi network without market. (b)(e) Distribution of the system liquidity at time $T=5,000$. Note that, y-scale is logarithmic. (e)(f) Distribution of the system liquidity at time $T=5,000$.

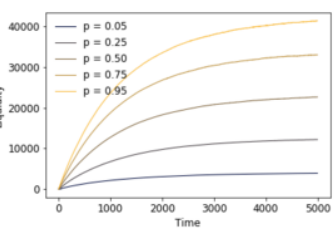

(a) $\sigma=0.05$

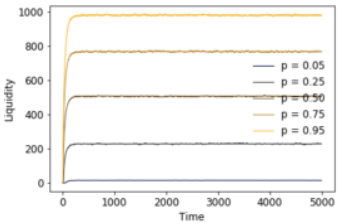

(d) $\sigma=0.3$

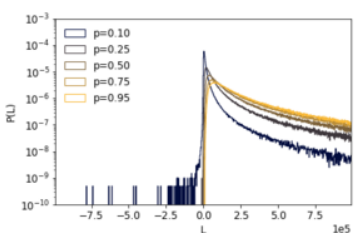

(b) $\sigma=0.05$

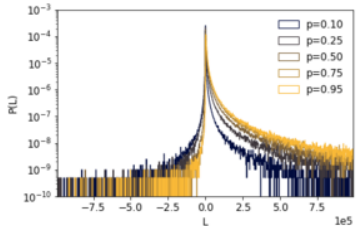

(e) $\sigma=0.3$

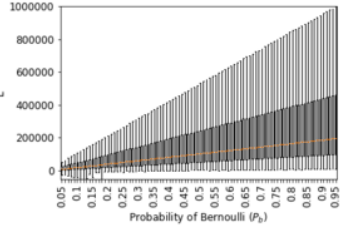

(c) $\sigma=0.05$

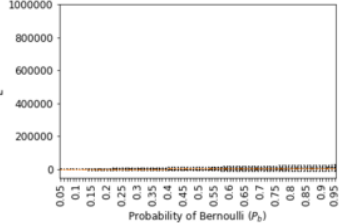

(f) $\sigma=0.3$

Figure 4. (a)(d) Median of the total liquidity at time $t$ for different combinations of Bernoulli probability $p$ for an Erdos-Renyi network with market. (b)(e) Distribution of the system liquidity at time $T=5,000$. Note that, y-scale is logarithmic. (e)(f) Distribution of the system liquidity at time $T=5,000$.

Similarly, the system reaches the steady state around $t=5,000$ for $\sigma=0.05$, whereas only 200 time-steps are needed for $\sigma=0.3$. Contrarily, we do not observe a decrease for lower activation rates in these cases. The inter-connectivity of the different individual nodes, makes this network more resilient to distress propagation.

As opposed to the cases discussed above, where the distribution of the overall liquidity value was symmetric around 0 , for the case of the Erdos-Renyi network with a market node, shown in Figures. 4.(b) and (e), we observe a right-tailed liquidity distribution. This asymmetry is greater for larger values of the Bernoulli probability $p$. However, differences observed for the different values of $p$ become smaller as the volatility increases. Both right and left tails flatten with increasing values of $\sigma$, whereas the peak increases, 
as was seen before. This asymmetry can also be observed in Figure. 4.(c) and (f), where boxplots show the distribution of liquidity for different activation probabilities.

\subsubsection{Power Law Network}

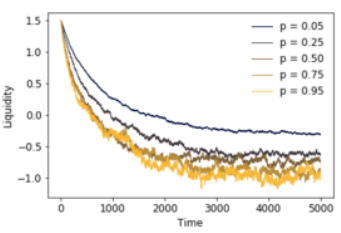

(a) $\sigma=0.05$

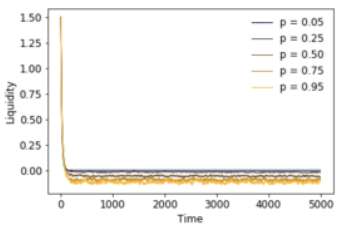

(d) $\sigma=0.3$

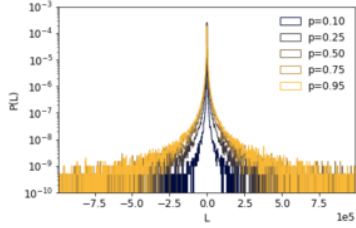

(b) $\sigma=0.05$

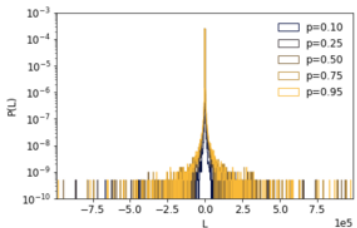

(e) $\sigma=0.3$

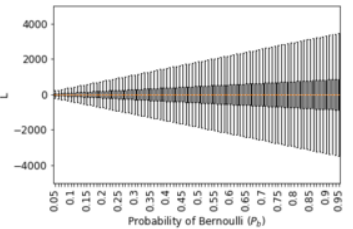

(c) $\sigma=0.05$

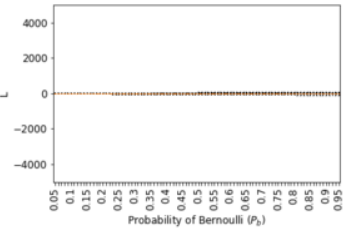

(f) $\sigma=0.3$

Figure 5. (a)(d) Median of the total liquidity at time $t$ for different combinations of Bernoulli probability $p$ for a Power Law network without market. (b)(e) Distribution of the system liquidity at time $T=1000$. Note that, y-scale is logarithmic. (e)(f) Distribution of the system liquidity at time $T=1000$.

Finally, we represent a capitalist economy using a Power Law network. For such network without the market node, we observe a decrease to a steady state for any value of p. Figure. 5 (a) and (d)) show the evolution of the median of the total liquidity in time for the different values of the Bernoulli probability $p$ and for different volatility values. We observe a faster decrease in the liquidity median over time as the higher is the volatility. Thus, for $\sigma=0.05$, the system reaches the steady state around $t=4,000$, whereas for $\sigma=0.3$ this happens around $t=200$. The distribution of liquidity at the steady state for different $p$ is still symmetric, as depicted in Figure. 5.(b) and (e). Moreover, we observe a similar behavior of the distribution of the liquidity as in the one node setting: for higher volatility, the peak of the distribution increases (around 1.5 in this case), whereas the tails have a bigger spread.

These new liquidity distributions are different because of the combination of the number of nodes and volatility. With 1,000 nodes, the aggregated Bernoulli probability of all nodes acts as a Binomial distribution, which occurs for both the earnings and payments. The spread of a Binomial distribution increases with $p$, as it can be observed in Figure. 5(c) and (f), which display the boxplots of the liquidity distribution across that variable. We can confirm this for $\sigma=0.05$ when the effect of volatility is very small. The spread of the liquidity is maximal for $p=1$, whereas for $p=0$ is minimal. For higher volatility, the multiplicative effect of $\sigma$ shapes the distribution of the liquidity, with a maximum in $p=0.5$. Therefore, we can conclude that the number of active edges matters concerning the range of the possible liquidity values.

In a Power Law network with 1,000 nodes, we have fewer edges than in an ErdosRenyi network with the same amount of nodes. The effect of this can be observed in Figure. 5(c) and (f), where the absolute amounts of liquidity are much lower than those 
observed for the Erdos-Renyi. Thus, the effect of the multiplicative noise $(\sigma)$ is also less significant, being the spread of the liquidity driven by the Binomial distribution. For this system, we have a liquidity ratio greater than 1 , but no liquidity from the market. This network acts at the end as an isolated system. The symmetry of the liquidity distribution is still present for any volatility.

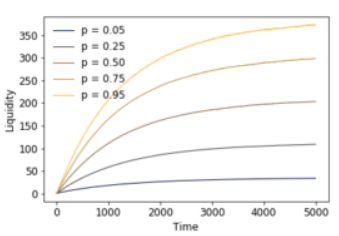

(a) $\sigma=0.05$

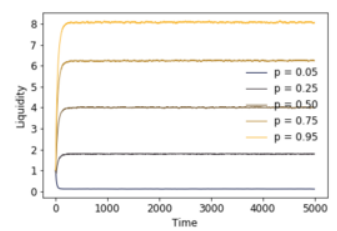

(d) $\sigma=0.3$

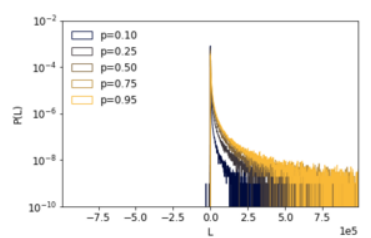

(b) $\sigma=0.05$

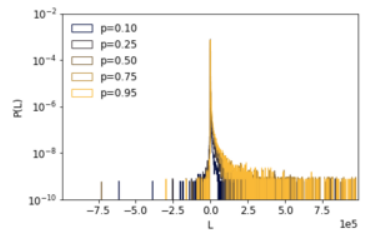

(e) $\sigma=0.3$

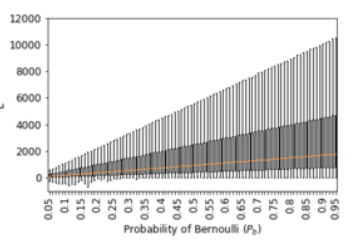

(c) $\sigma=0.05$

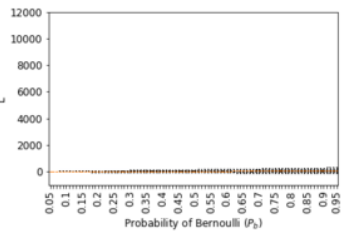

(f) $\sigma=0.3$

Figure 6. (a)(d) Median of the total liquidity at time $t$ for different combinations of Bernoulli probability $p$ for a Power Law network with market. (b)(e) Distribution of the system liquidity at time $T=1000$. Note that, y-scale is logarithmic. (e)(f) Distribution of the system liquidity at time $T=1000$.

When analyzing the results for the case where we include the market node, we again observe a growth for the liquidity median of all nodes. The evolution of the median of the liquidity of all nodes is shown in Figure. 6.(a) and (d), where we observe a faster increase in the liquidity median over time as higher is the volatility. As for a Power Law without a market node, for $\sigma=0.05$, the system reaches the steady state around $t=4,000$, whereas for $\sigma=0.3$ this happens around $t=200$. Figure. 6.(b) and (e) depicts an asymmetry of the distribution of liquidity at the steady state for different values of $p$ caused by the presence of the market node. The spreads are smaller due to the fewer number of edges between nodes in the Power Law network. Both right and left tails flatten as higher is the $\sigma$, whereas the peak increases, as it was seen before. Despite the effect of the market node, the spread of the liquidity for a Power Law is still driven by the Binomial distribution, and volatility has a smaller consequence compared to the ErdosRenyi network. In Figure. 6.(c) and (f), the alternative representation of the distribution of liquidity at the steady state with boxplots confirms the asymmetry for any volatility.

\section{Conclusions}

In this article, we have studied how the risk of default propagates in different synthetic financial networks by using a liquidity model. Networks are a valid representation of economic transactions such as wires to pay for services or goods. Based on previous works on wealth distribution, the used model combines two stochastic terms: an additive noise accounting for the capability of trading and paying obligations, regulated by a parameter 
$p$, and a multiplicative noise representing the variations of the market, controlled by a parameter $\sigma$.

Our results show that, even in the presence of large noise values, network dynamics reach a steady-state, with the only exception of the Erdos-Renyi network without market node. In this sense, the presence of a market node seems crucial for the stability of the system. The fact that a market node injects money to nodes constantly causes an increase in the median liquidity. In contrast, when there isn't such a market node, the total system liquidity remains constant for any $p$. For this reason, we do not observe significant differences between the behavior for low and high Bernoulli probability $(p)$ where there is a network without the market. Therefore, in that case, the liquidity ratio and the volatility $(\sigma)$ control the amount of money the nodes can accumulate. In spite of the median liquidity distribution being comparable for all the networks whenever a market node is included, differences arise in the absence of this node. This is, we detect a network effect when there is no market node. When this happens, the different connections between nodes due to the different network topologies have a bigger impact. We conclude that inter-connectivity is crucial for the time evolution of liquidity.

From an economic perspective, the network structure of economic interactions and its combination with the system volatility $(\sigma)$ is crucial to determine the maximum default probability. We conclude that this model can be a valuable tool to study systemic risks of different economic systems. In this sense, Artificial Intelligence may help us to understand the behavior of Financial Networks. Future research following this work should focus on studying strategies to prevent risk of default and cascade effects in the different settings.

In addition, further research should also study the effect of an optimal rewiring of nodes from an agent-based perspective, to design protocols that ensure resilience against disruption propagation.

\section{References}

[1] E. Atalay, A. Hortasu, J. Roberts, and C. Syverson. Network structure of production. Proceedings of the National Academy of Sciences (PNAS), 108(12):5199-5202, 2011.

[2] A. Barja, A. Martínez, A. Arenas, P. Fleurquin, J. Nin, J. Ramasco, and E. Tomás. Assessing the risk of default propagation in interconnected sectoral financial networks. EPJ Data Science, 8(1):32, 2019.

[3] F. Corsi, F. Lillo, D. Pirino, and L. Trapine. Measuring the propagation of financial distress with grangercausality tail risk networks. Journal of Financial Stability, 38:18-36, 2018.

[4] C. Bode and S.M. Wagner. Structural drivers of upstream supply chain complexity and the frequency of supply chain disruptions. Journal of Operations Management, 36:215-228, 2015.

[5] K Zhao, Z Zuo, and JV Blackhurst. Modelling supply chain adaptation for disruptions: An empirically grounded complex adaptive systems approach. J. of Operations Management, 65(2):190 212, 2019.

[6] L. Veraart. Distress and default contagion in financial networks. Math. Finance, 30:705-737, 2020.

[7] E. Letizia and F. Lillo. Corporate payments networks and credit risk rating. EPJ Data Sci., 8(21), 2019.

[8] J. Bouchaud and M. Mézard. Wealth condensation in a simple model of economy. Phisica A, 282:536545, 2000 .

[9] J. Nin, B. Salbanya, P. Fleurquin, E. Tomas, A. Arenas, and J. Ramasco. Modelling financial distress propagation on customer-supplier networks. Chaos Journal, 31(5), 2021. 